\title{
WISE 2007 Extended Best Papers
}

\author{
Boualem Benatallah • Fabio Casati • \\ Dimitrios Georgakopoulos • Claude Godart
}

Received: 26 November 2008 / Accepted: 28 November 2008 /

Published online: 8 January 2009

(C) Springer Science + Business Media, LLC 2009

\section{Guest editorial: Special issue on web information systems engineering}

Dear colleagues and readers, welcome to the WWWJ special issue on the WISE 2007 conference which took place in Nancy, France, in December 2007.

Overall the conference had nearly 200 submissions. Out of these, we selected 40 full-papers (acceptance rate $20 \%$ ) and 18 short papers (acceptance rate $9 \%$ ) for conference presentations, and out of these we selected a shortlist of top papers to be submitted to the special issue and to go through another round of evaluation and review. In the end we selected five papers that are of excellent quality and that, we believe, cover a nice range of topics in the space of Web information systems.

The first two papers deals with the problems of selecting and customizing services and user interfaces. The paper "Rater Credibility Assessment in Web Services Interactions", by Zaki Malik and Athman Bouguettaya, shows how to select web services based on trust parameters, and specifically by leveraging reputation metrics and protecting these metrics from malicious raters. In "Customising Web Information Systems According to User Preferences", Klaus-Dieter Schewe, Bernhard

\footnotetext{
B. Benatallah

The University of New South Wales, Sydney, Australia e-mail: boualem@cse.unsw.edu.au

F. Casati

University of Trento, Trento, Italy

e-mail: fabio.casati@gmail.com

D. Georgakopoulos

CSIRO, Canberra, Australia

e-mail: dimitrios.georgakopoulos@csiro.au

C. Godart $(\bowtie)$

Nancy University, Nancy, France

e-mail: claude.godart@loria.fr
} 
Thalheim, and Qing Wang present a theoretical model for the customization of Web information systems, based on user preferences and constraints. The model is based on the co-design approach to Web development, but is applicable to a variety of other Web design methodologies.

In "BEIRA: An area-based user interface for map service", by Osamu Masutani and Hirotoshi Iwasaki, the authors propose a way to simplify browsing maps by means of what is called geo-semantic clustering, that groups information based on location and semantics of the places being searched to provide high-level summaries of the characteristics of an area.

The next paper is a direct result from an empirical study. In "A Domain-Driven Approach for Detecting Event Patterns in E-Markets", by Piyanath Mangkorntong and Fethi Rabhi, an event processing system (EPS) architecture is proposed, driven by experiences with e-markets, seen as event generating systems. Mangkorntong and Rabhi discuss strengths and weaknesses of commercial EPSs and show how strengths can be leveraged by combining EPSs.

Finally, in "Independence of Containing Patterns Property and its Application in Tree Pattern Query Rewriting Using Views" by Junhu Wang, Jeffrey Xu Yu, and Chengfei Liu, dives deep into the database side of Web information system and specifically presents a study that allows, among other benefits, to optimize the views to be cached and more in general to optimize view design in information integration systems.

We sincerely hope you enjoy reading these papers as much as we did and we look forward to meeting you at the forthcoming editions of the WISE conference. 